\title{
Analysis According to Characteristics of 18 Cases of Brachial Plexus Tumors : A Review of Surgical Treatment Experience
}

\author{
In-Ho Jung, M.D., Kyeong-Wook Yoon, M.D., Young-Jin Kim, M.D., Ph.D., Sang Koo Lee, M.D., Ph.D. \\ Department of Neurosurgery, Dankook University College of Medicine, Cheonan, Korea
}

Objective : Because the anatomical structure of the brachial plexus is very complex, surgical treatment of tumors in this region is challenging. Therefore, a lot of clinical and surgical experience is required for successful treatment; however, many neurosurgeons have difficulty accumulating this experience owing to the rarity of brachial plexus tumors. The purpose of this report is to share our surgical experience with brachial plexus tumor with other neurosurgeons.

Methods : The records of 18 consecutive patients with brachial plexus tumors who underwent surgical treatment between January 2010 and December 2017 in a single institution were retrospectively reviewed. The surgical approach was determined according to the tumor location and size, and intraoperative neurophysiological monitoring (IONM) was used in most of cases to prevent iatrogenic nerve injury during surgery. In addition, to evaluate the differences in tumor characteristics according to pathologic diagnosis, the tumors were divided twice into two groups, based on two separate classifications, and statistical analysis was performed.

Results : The 18 brachial plexus tumors comprised 15 (83.3\%) benign peripheral nerve sheath tumors including schwannoma and neurofibroma, one (5.6\%) malignant peripheral nerve sheath tumor, one (5.6\%) benign tumor of non-neural sheath origin (neurogenic cyst), and one (5.6\%) metastatic tumor (papillary carcinoma). The authors analyzed relationship between tumor size/ location and tumor characteristic parameters such as age, size, right-left, and pathology. There were no statistically significant differences except a tendency of bigger tumor size in young age.

Conclusion : For a successful surgical outcome, an appropriate surgical approach is essential, and the appropriate surgical approach is determined by the location and size of the tumor. Furthermore, applying IONM may prevent postoperative complications and it is favorable option for brachial plexus tumors surgery.

Key Words : Brachial plexus neuropathy · Nerve sheath neoplasms · Monitoring, Intraoperative.

\section{INTRODUCTION}

Brachial plexus tumors (BPTs) may cause neurological symptoms such as palpable masses or localized pain ${ }^{9}$. Howev- er, because BPTs are relatively rare, neurosurgeons have difficulty in surgery because of an insufficient clinical and surgical experience with $\mathrm{BPTs}^{4}$. In addition, other published reports are mostly case reports or small-sized studies ${ }^{1,4,7)}$. Therefore,

- Received : March 1, 2018 •Revised : May 21, 2018 •Accepted : June 21, 2018

- Address for reprints : Young-Jin Kim, M.D., Ph.D.

Department of Neurosurgery, Dankook University College of Medicine, 201 Manghyang-ro, Dongnam-gu, Cheonan 31116, Korea

Tel : +82-41-550-6280, Fax : +82-41-552-6870, E-mail : melway@hanmail.net

This is an Open Access article distributed under the terms of the Creative Commons Attribution Non-Commercial License (http://creativecommons.org/licenses/by-nc/4.0) which permits unrestricted non-commercial use, distribution, and reproduction in any medium, provided the original work is properly cited. 
there is less opportunity for direct and indirect experience with BPTs, and it is necessary for neurosurgeons in the field to accumulate experience with treatment of BPTs. In the present study, to share our experiences with the treatment of BPTs, we collected and analyzed the cases of patients who underwent surgical treatment with BPT in a single institution for eight years.

\section{MATERIALS AND METHODS}

\section{Patient population}

All patients presented to a single institution with BPT and underwent surgical treatment between January 2010 and December 2017. The authors reviewed the medical records of all 18 patients retrospectively and collected data including the age, sex, clinical presentation, tumor characteristics, and pathology. Magnetic resonance imaging (MRI) studies were performed in all patients to evaluate tumor size, location, and characteristics.

\section{Surgical approach and procedure}

In this study, when the BPT was located from cord level to division level, the authors applied an anterior supraclavicular approach. When the tumor was located below the cord level, the authors applied an anterior infraclavicular approach. A supra\&infraclavicular approach was used in case tumor was large enough to need both approaches due to clavicle. The patient was positioned supine with a roll under the ipsilateral shoulder and the head turned 30 degrees toward the opposite side. For a supraclavicular approach, a V-shaped skin incision was made, with one side parallel to the clavicle and one side parallel to the sternocleidomastoid muscle (SCM). When the platysma was exposed, it was incised, with dissection along the lateral border of the SCM (Fig. 1A). When the omohyoid muscle is seen crossing obliquely, it can be retracted or cut (Fig. 1B). After the omohyoid muscle was cut, the phrenic nerve could be visualized in front of the anterior scalene muscle. This nerve should be preserved (Fig. 1C). The brachial plexus was somewhat exposed, but because the BPT was large and crossed the clavicle, an infraclavicular approach and clavicle resection were reqired. An additional incision was made over the deltoid-pectoral groove. After dissection of the deltoid muscle and the pectoralis major muscle, the pectoralis minor muscle and its origin at the coracoid process were encountered. With retraction of the pectoralis minor muscle upward and downward, the cord level of the brachial plexus was visible (Fig. 1D). The entire tumor was exposed, displacing the normal elements of the nerve axon to one side, and cautious dissection was performed. The tumor was removed en bloc (Fig. 1E and F). After BPT removal, the entire brachial plexus anatomy could be seen (Fig. 1G). The encapsulated tumor had high vascularity, yellowish color, and hardness (Fig. 1H).
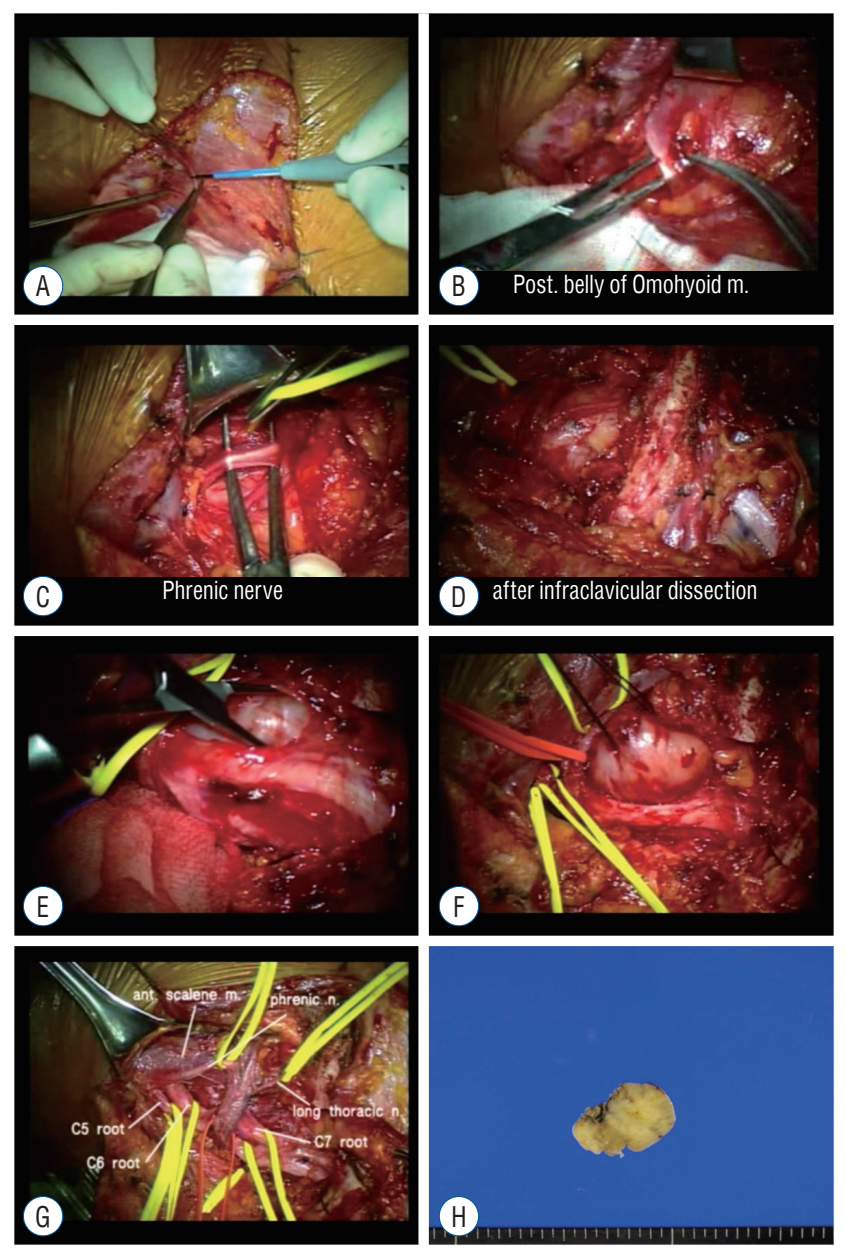

Fig. 1. Intraoperative images of brachial plexus tumor removal. A : Sternocleidomastoid muscle is exposed after skin incision. B : The omohyoid muscle is dissected and cut. C : Phrenic nerve in front of the anterior scalene muscle can be seen. D : After supra \& infraclavicular approach, the clavicle traverses the middle of the tumor and interferes with tumor dissection. $\mathrm{E}$ and $\mathrm{F}$ : Brachial plexus tumor is dissected and removed. G : After the brachial plexus tumor removal, entire brachial plexus anatomy can be seen. $\mathrm{H}$ : Gross photograph of brachial plexus tumor, which has high vascularity, yellowish color and hard character. 


\section{Intraoperative neurophysiological monitoring (IONM)}

The authors checked preoperative electromyography (EMG) to measure baseline evoked potential. In general anesthesia, short-acting muscle relaxants (rocuronium bromide) are given for intubation, since then, muscle relaxants have not been injected. Also, the surgeon confirmed recovery from effect of the muscle relaxants by using stimulus testing. Multiple EMG needles are placed into the muscles which the surgeon want to examined. The authors usually use motor evoked potential (mEP), somatosensory evoked potential (ssEP) and direct nerve stimulation for brachial plexus tumor. After stimulation of the peripheral nerve at the upper extremity, ssEP was recorded from the transcranial electrode. The stimulus was given automatically every 15 minutes (single stimuli of $200 \mu \mathrm{sec}$ duration, intensity $25 \mathrm{~mA}$, stimulation rate $2.31 \mathrm{~Hz}$ ). mEPs were evoked by using transcranial electrical simulation with multi-pulse or train stimulation technique, and recorded from the target muscle. Direct nerve stimulation was used to make sure when the structure was uncertain whether it was nerve or not, by bipolar stimulation forceps intraoperatively. It would help to decide whether suspicious neural structure could be sacrificed or should be preserved.

\section{Analysis method}

For statistical analysis, we used the R statistical software program (R version 3.2.4, Comprehensive R Archive Network, http://cran.r-project.org). Eighteen cases of brachial plexus tumor were divided into two groups by its size. 'Large group' was defined as a tumor size greater than $3 \mathrm{~cm}$, and 'Small group' was defined as a tumor size less than $3 \mathrm{~cm}$. MannWhitney U test and Fisher's exact test was performed to determine statistical significance with large group and small group, and $p \leq 0.05$ was set as the threshold. The authors analyzed also, characters of brachial plexus tumor according to tumor location and surgical approach. All tumors were classified into three groups by its location as follow : infraclavicular group, supraclavicular group, and supra \& infraclavicular group.

\section{RESULTS}

\section{Patient population \& clinical presentation}

A total 18 of patients with BPTs were enrolled. The study included 8 men (44.4\%) and 10 women (55.6\%), and the median age of the population was 51 years (interquartile range [IQR], 24-80 years). Demographic data and characteristics of the patients are detailed in Table 1 . Almost all patients with BPT (17 patients, 94.4\%) had at least one symptom. The most common symptom was a palpable mass, which was present in 17 patients (94.4\%). The next most common symptoms were localized tenderness (nine patients, 55.6\%), sensory alterations (eight patients, 44.4\%), and motor weakness (four patients, $22.2 \%$ ). Sensory alterations included anesthesia, hypesthesia, hyperesthesia, and dysesthesia. Clinical symptoms of the patients are detailed in Table 2.

Table 1. Demographic data and brachial plexus tumor characteristics

\begin{tabular}{lc}
\hline Variable & Value $(\mathbf{n}=\mathbf{1 8})$ \\
\hline Age & $51(24-80)$ \\
Male & $8(44.4)$ \\
Female & $10(55.6)$ \\
Right & $8(44.4)$ \\
Left & $10(55.6)$ \\
Pathology & \\
Schwannoma & $11(61.1)$ \\
Neurofibroma & $4(22.2)$ \\
Neurogenic cyst & $1(5.6)$ \\
Papillary carcinoma & $1(5.6)$ \\
MPNST & $1(5.6)$ \\
Approach & $10(55.6)$ \\
Supraclavicular & $6(33.3)$ \\
Infraclavicular & $2(11.1)$ \\
Supra \& infraclavicular & \\
\hline
\end{tabular}

$\overline{\text { Values are presented as mean (range) or number (\%). MPNST : malignant }}$ peripheral nerve sheath tumor

Table 2. Clinical symptoms of 18 patients with brachial plexus tumor

\begin{tabular}{lc}
\hline Symptoms & Value \\
\hline Asymptomatic & $1(5.6)$ \\
Symtomatic & $17(94.4)$ \\
Palpable mass & $17(94.4)$ \\
Localized tenderness & $9(55.6)$ \\
Sensory alterations & $8(44.4)$ \\
Motor weakness & $4(22.2)$ \\
\hline
\end{tabular}

Values are presented as number (\%) 


\section{Tumor characteristics analysis}

Eight patients presented with right-sided BPT (44.4\%), and 10 patients (55.6\%) had left-sided BPT. In this study, the aouthos categorized BPT to three groups. First, benign peripheral nerve sheath tumor (PNST), including schwannoma and neurofibroma, was present in 15 patients (83.3\%). Second, malignant PNST was present in one patient (5.6\%). Third, benign tumors of non-neural sheath origin (neurogenic cyst) were present in one patient (5.6\%). Metastatic tumor, including papillary carcinoma, was present in one patient (5.6\%). The most common location was trunk level in 10 cases (55.6\%). A large tumor may occupy two or more levels. The summary of all patients is presented in Table 3 .

To analysis character of brachial plexus tumor according to size, all tumors were classified into two groups. There were no statistically significant differences in sex, right-left, pathology, and approach between large group and small group. However, the mean age of the large group was 45 years, and the mean age of the small group was 64 years. It is tend to occur larger tumor in young age with statistical significance $(p=0.046)$. It is presented in Table 4 . Also, the authors analyzed relationship between tumor location and tumor characteristic parameters such as age, size, right-left, and pathology. There were no statistically significant differences and it is presented in Table 5.

\section{Clinical outcomes}

Gross tumor removal was possible in 17 of the 18 patients. The mean postoperative follow-up period was 19.8 months. Localized tenderness was improved after surgery in nine out of nine patients. Hypesthesia and motor weakness were also improved after surgery, but the improvement of these symptoms took several weeks to several months. Hypesthesia and motor weakness newly occurred in one patient because surgical dissection was difficult owing to severe adhesion. Motor weakness of shoulder abduction and elbow flexion with a motor grade 4 occurred immediate postoperatively, but gradually improved, and it was recovered after 2 months from surgery.

\section{Case presentation 1 (No. 10)}

Fifty seven years old male patient who complained of a left supraclavicular area palpable mass was referred from department of head and neck surgery. Brachial plexus MRI revealed

Table 3. Summary of 18 patients with brachial plexus tumor

\begin{tabular}{|c|c|c|c|c|c|c|c|c|c|}
\hline Case No. & Age & Sex & Size $(\mathrm{cm})$ & $R / L$ & Location & Extent of resection & Pathology & Approach & IONM \\
\hline 1 & 80 & $\mathrm{~F}$ & $2.5 \times 2.0 \times 2.4$ & $\mathrm{R}$ & Rt. axillary area, branch & GTR & Schwannoma & Infraclavicular & + \\
\hline 2 & 60 & M & $3.0 \times 2.7 \times 5.5$ & L & Lt. upper trunk \& division & GTR & Papillary carcinoma & Supraclavicular & - \\
\hline 3 & 33 & M & $3.5 \times 4.2 \times 3.0$ & $\mathrm{~L}$ & Lt. terminal branches & GTR & Schwannoma & Infraclavicular & + \\
\hline 4 & 43 & $\mathrm{~F}$ & $7.3 \times 5.3 \times 8.6$ & L & Lt. middle lower trunk & GTR & Neurofibroma & Supraclavicular & + \\
\hline 5 & 24 & $\mathrm{~F}$ & $9.3 \times 5.0 \times 5.2$ & $L$ & Lt. terminal branches & GTR & Neurogenic cyst & Infraclavicular & + \\
\hline 6 & 75 & $\mathrm{~F}$ & $4.0 \times 3.3 \times 4.0$ & $L$ & Lt. ant. division & GTR & Schwannoma & Supraclavicular & + \\
\hline 7 & 59 & M & $2.0 \times 2.0 \times 2.6$ & $\mathrm{R}$ & Rt. ant. division & GTR & Schwannoma & Supraclavicular & + \\
\hline 8 & 66 & M & $2.0 \times 2.0 \times 2.0$ & L & C5 root \& upper trunk & GTR & Schwannoma & Supraclavicular & + \\
\hline 9 & 36 & M & $5.2 \times 3.2 \times 2.9$ & R & $\mathrm{C} 8, \mathrm{~T} 1$ root \& lower trunk & GTR & Schwannoma & Supraclavicular & + \\
\hline 10 & 57 & M & $4.0 \times 3.0 \times 2.0$ & L & Upper trunk & STR & MPNST & Supraclavicular & + \\
\hline 11 & 58 & $\mathrm{~F}$ & $3.0 \times 2.7 \times 1.7$ & $L$ & Upper trunk & GTR & Schwannoma & Supraclavicular & + \\
\hline 12 & 62 & $\mathrm{~F}$ & $6.2 \times 3.7 \times 5.1$ & $\mathrm{R}$ & Cord & STR & Neurofibroma & Infraclavicular & + \\
\hline 13 & 46 & $\mathrm{~F}$ & $4.8 \times 3.5 \times 2.2$ & R & C6, C7 root \& trunk & GTR & Schwannoma & Supraclavicular & + \\
\hline 14 & 42 & $\mathrm{~F}$ & $3.9 \times 2.4 \times 1.9$ & R & Upper \& middle trunk & GTR & Schwannoma & Supraclavicular & + \\
\hline 15 & 44 & M & $1.2 \times 1.8 \times 2.1$ & L & Lt. terminal branch & GTR & Neurofibroma & Infraclavicular & + \\
\hline 16 & 45 & M & $11.6 \times 3.0 \times 3.1$ & $\mathrm{R}$ & C7 root \& middle trunk & GTR & Schwannoma & Supra \& infraclavicular & + \\
\hline 17 & 64 & $\mathrm{~F}$ & $1.0 \times 1.1 \times 0.9$ & $\mathrm{R}$ & Post. cord & GTR & Schwannoma & Infraclavicular & + \\
\hline 18 & 31 & $\mathrm{~F}$ & $4.6 \times 3.3 \times 4.7$ & $L$ & Middle trunk & GTR & Neurofibroma & Supra \& infraclavicular & + \\
\hline
\end{tabular}

$\mathrm{R}$ : right, L : left, IONM : intraoperative neurophysiological monitoring, F : female, Rt. : right, GTR : grossly total removal, $M$ : male, Lt. : left, ant. : anterior, STR : subtotal removal, MPNST : malignant peripheral nerve sheath tumors, post. : posterior 
Analysis of Brachial Plexus Tumors | Jung IH, et al.

Table 4. Relationship between tumor characteristics and size

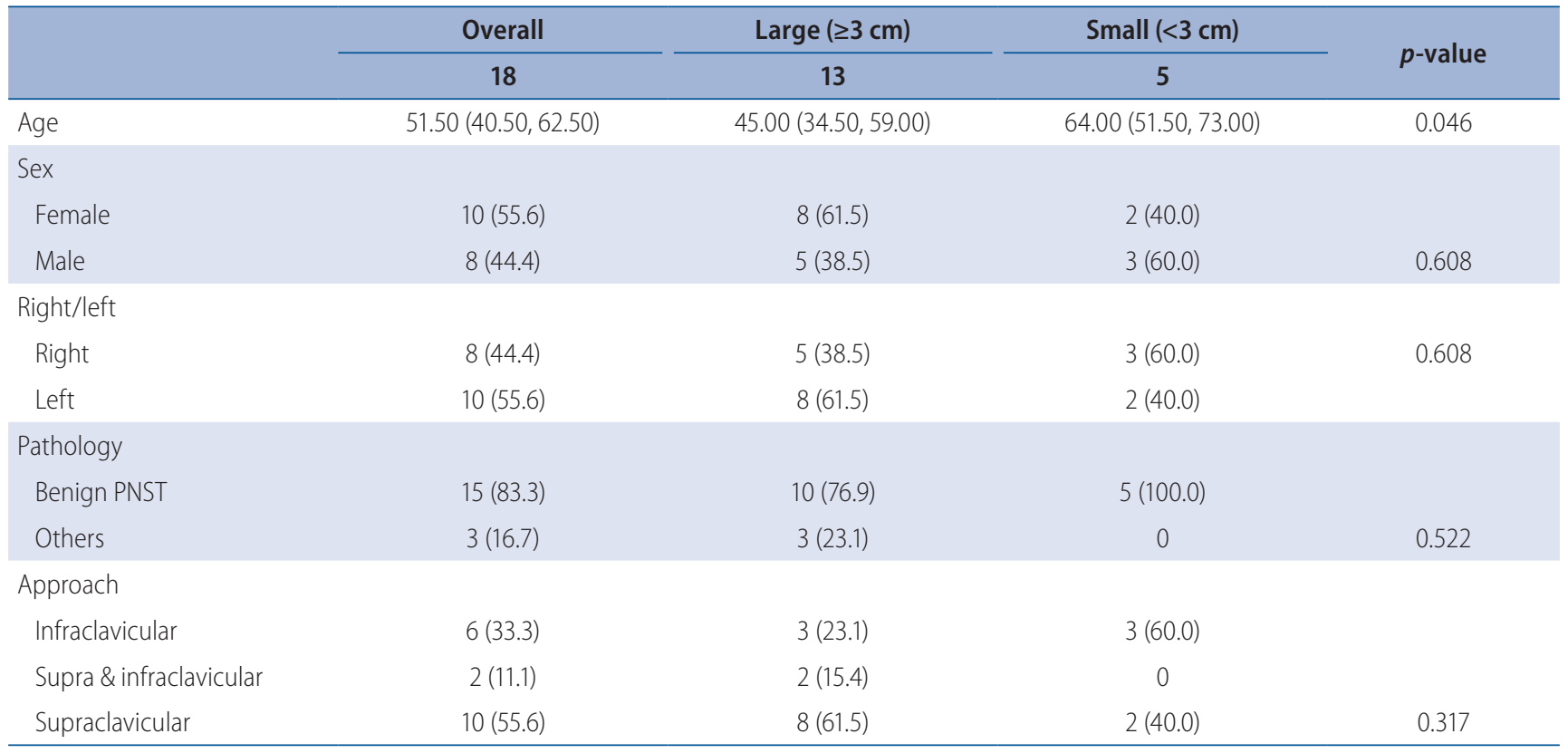

Values are presented as median (interquartile range ) or number (\%). PNST : peripheral nerve sheath tumor

Table 5. Analysis according to tumor location \& surgical approach

\begin{tabular}{|c|c|c|c|c|c|}
\hline & Overall & Infraclavicular & Supra \& infraclavicular & Supraclavicular & $n$-value \\
\hline & 18 & 6 & 2 & 10 & \\
\hline Age & $51.50(42.25,61.50)$ & $53.00(30.75,68.00)$ & $38.00(31.00,-)$ & $57.50(42.75,61.50)$ & 0.438 \\
\hline Size & $51.50(42.25,61.50)$ & $3.350(1.850,6.975)$ & $7.850(4.700,-)$ & $4.000(2.900,5.275)$ & 0.354 \\
\hline \multicolumn{6}{|l|}{ Sex } \\
\hline Female & $10(55.6)$ & $4(66.7)$ & $1(50.0)$ & $5(50.0)$ & \\
\hline Male & $8(44.4)$ & $2(33.3)$ & $1(50.0)$ & $5(50.0)$ & 0.808 \\
\hline \multicolumn{6}{|l|}{ Right/left } \\
\hline Right & $8(44.4)$ & $3(50.0)$ & $1(50.0)$ & $4(40.0)$ & 1.000 \\
\hline Left & $10(55.6)$ & $3(50.0)$ & $1(50.0)$ & $6(60.0)$ & \\
\hline \multicolumn{6}{|l|}{ Pathology } \\
\hline Benign PNST & $15(83.3)$ & $5(83.3)$ & $2(100.0)$ & $8(80.0)$ & \\
\hline Others & $3(16.7)$ & $1(16.7)$ & 0 & $2(20.0)$ & 1.000 \\
\hline
\end{tabular}

Values are presented as median (interquartile range ) or number (\%). PNST : peripheral nerve sheath tumor

lobulated contrast enhancing large mass in left trunk to divisional portion. The tumor was suspected as malignancy, because of lymph node enlargement and adhesion with nearby tissue, vessel, or nerve (Fig. 2A). Surgical resection by supraclavicular approach with IONM was scheduled. Subtotal tumor removal was achieved without any postoperative complication. Postoperative MRI showed subtotal resection was achieved (Fig. 2B). Pathologic results revealed that the tumor consisted with neurofibrosarcoma. However, the patient complained of radiating pain on left upper extremity again after 6 months from surgery. Unfortunately, the brachial plexus MR showed recurred neurofibrosarcoma (Fig. 2C). The authors suggested revision surgery, but the patient refused and transferred to another hospital. 


\section{Case presentation 2 (No. 16)}

The case of a 45 -year-old male who presented with a tingling sensation in the right finger and a palpable mass in the right supraclavicular area visited to department of neurosugery. Brachial plexus MRI revealed a well-defined contrast-enhancing multilobulated large mass extending from the $\mathrm{C} 7$ nerve root to the middle trunk (Fig. 3). EMG result showed findings suggestive of plexopathy of the right lower brachial plexus. Surgical resection with a supra- and infraclavicular approach with IONM was scheduled. The pathologic study showed biphasic pathologic pattern which implied schwannoma (Fig. 4). Six months after the surgery, the patient reported no definite neurological deficits, and his initial presenting symptom, a tingling sensation of right fin- ger, had disappeared.

\section{DISCUSSION}

Symptoms caused by brachial plexus tumors present in a wide variety of ways. Lwu and Midha" described symptoms of brachial plexus tumors, based on history and physical examination. Patients were reported to complain of sensory alterations, painless lumps, pain, motor deficits, or autonomic dysfunction. As the results of our study show, the three most common symptoms of brachial plexus tumors are palpable mass, localized tenderness, and sensory alteration. These
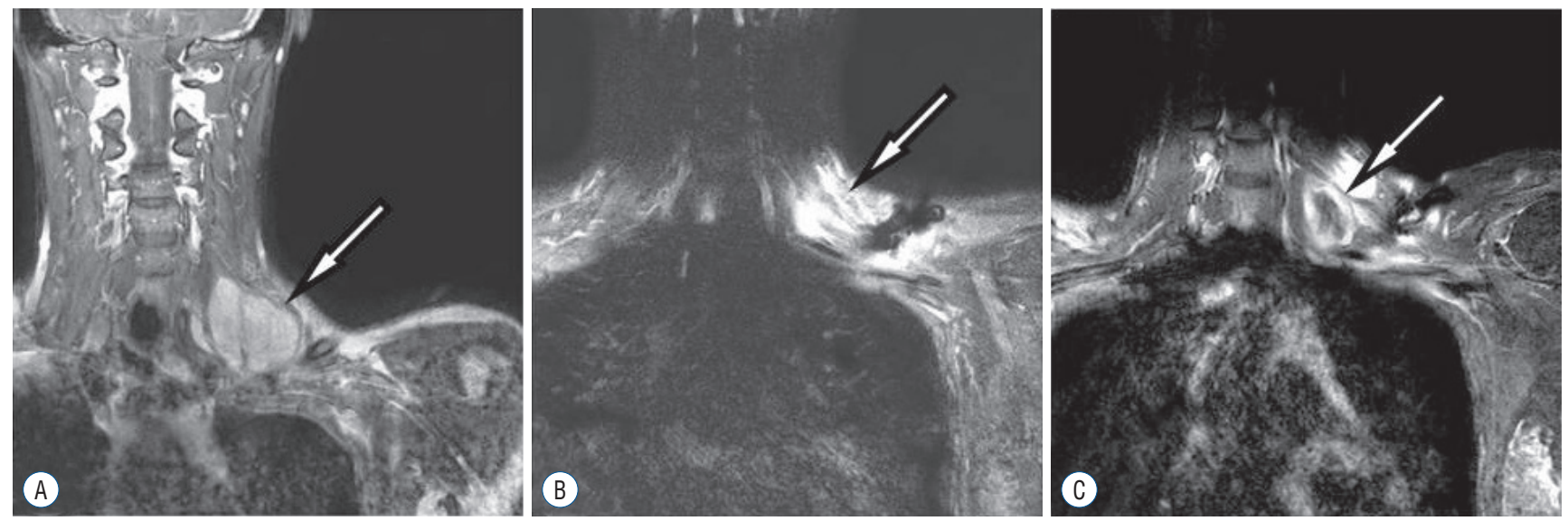

Fig. 2. A : Preoperative T1 weighted enhancing MR imaging shows homogenous enhancing mass (white arrow). B : Postoperative MR imaging shows subtotal removal of tumor (white arrow). C : MR imaging after 6 months from surgery shows recurrence of tumor (white arrow). MR : magnetic resonance.
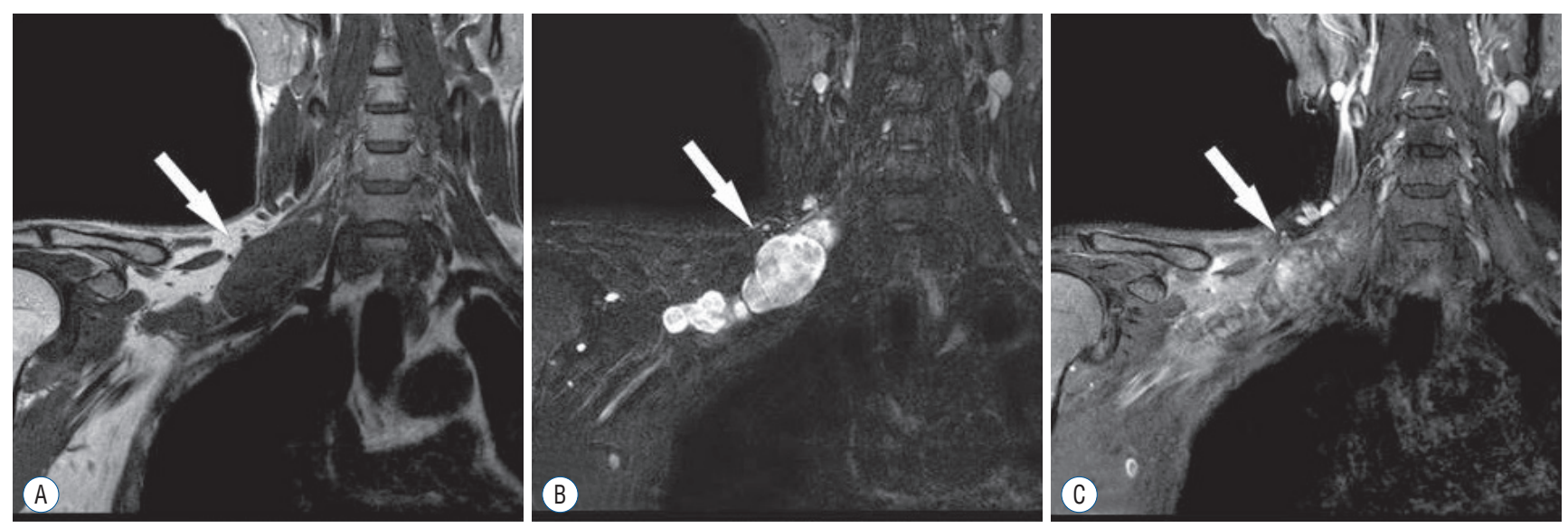

Fig. 3. Brachial plexus magnetic resonance imaging revealed a well-defined contrast enhancing multi-lobulated large mass from C7 nerve root to middle trunk (white arrows). A : Coronal T1 weighted image shows the intermediate signal of brachial plexus tumor. B : Coronal T2 weighted short tau inversion recovery image shows the high signal of brachial plexus tumor. C : Coronal T1 with enhancement shows brachial plexus tumor with heterogeneous enhancing. 


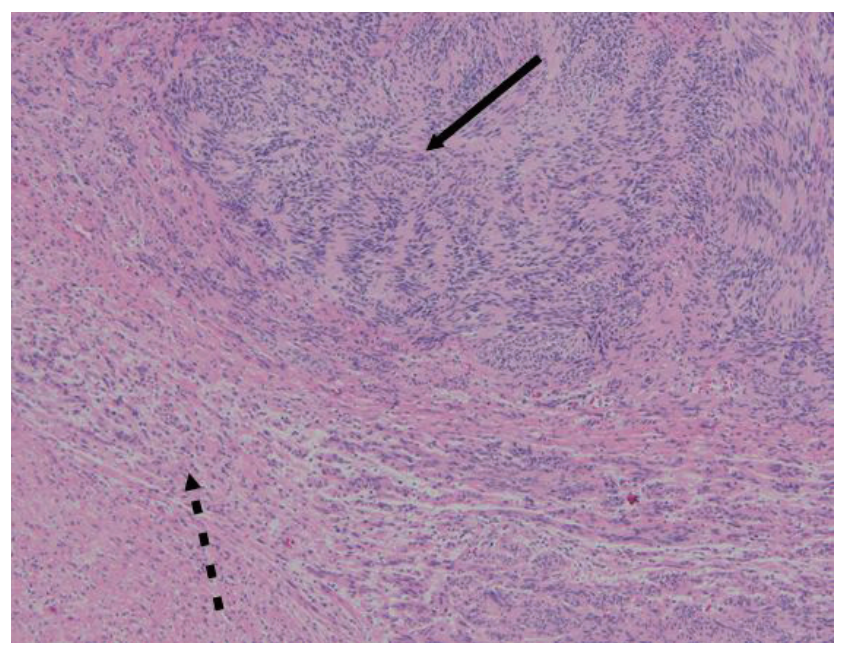

Fig. 4. In microscopic finding with hematoxylin and eosin (H\&E) stain and original magnification $\times 100$, compact hypercellular Antoni $A$ areas (solid arrow) and myxoid hypocellular Antoni B areas (dotted arrow) are coexist. And this is typical pathologic finding of schwannoma.

symptoms are so subtle that diagnosis can be difficult without detailed physical examinations. If the pathologic diagnosis of the tumor is neurofibroma, café-au-lait macules, Lisch nodules in the iris, dermal neurofibromas, and axillary or inguinal freckling can be observed in the skin. Therefore, clinicians should also be attentive to cutaneous features"

The surgical approach is determined according to the location and size of the BPT. Das et al. ${ }^{4)}$ reported that an anterior supraclavicular approach is suitable for tumors involving the roots and trunks. An anterior infraclavicular approach is suitable for lesions involving the cords and distal plexus elements, and a posterior subscapular approach is suitable for tumors involving the proximal roots ${ }^{4}$. The posterior subscapular approach has some advantages over the classical anterior approach. The biggest advantage of the posterior subscapular approach is that it can provide excellent exposure of the spinal roots at very proximal levels, as well as the intraforaminal portion of spinal nerves ${ }^{3,5}$. However, we did not utilize the posterior subscapular approach in our 18 patients, for the following reasons. First, there were no BPTs located at the very proximal root level among our cases. Second, the posterior approach includes troublesome and time-consuming tasks such as rib resection. Lastly, the authors were unfamiliar with this approach. The size, as well as the location, of the tumor has a great influence on the decision regarding surgical approach $^{10)}$. When the size of the tumor is too large to perform en bloc removal, or when the tumor is large and passes under the clavicle, resection of the clavicle can be choose for a suprainfraclavicular approach. The authors applied a supraclavicular approach in 10 patients (55.6\%), an infraclavicular approach in six patients (33.3\%), and a supra-infraclavicular approach in two patients (11.1\%).

It is widely accepted that BPT can be divided into five major categories. The first category is PNST, which includes schwannoma, neurofibroma, and malignant peripheral nerve sheath tumor. The second is benign tumor of non-neural sheath origin, including desmoid, ganglioneuroma, meningioma, and lipoma. The third category is tumor of vascular origin, including hemangiopericytomas. The fourth category is localized hypertrophic neuropathy, and the fifth is metastatic tumor ${ }^{8)}$. Previous studies have shown that the most common primary nerve tumor is PNST, including schwannomas and neurofibromas. Other categories of tumor, with the exception of PNST, have a relatively low incidence rate ${ }^{2,6)}$. Binder et al. ${ }^{1)}$ reported 25 cases of BPT in 2004, among which there were 20 benign PNSTs (80\%), 4 malignant PNSTs (16\%), and 1 nonneural sheath origin tumor (4\%). Compared to our study, the ratio of benign PNSTs did not differ greatly, while the proportion of malignant PNSTs was relatively higher.

The authors divided tumor into two groups according to size. There were no statistically significant differences in sex, right-left, pathology, and approach between large group and small group. However, the mean age of the large group was 45 years, and the mean age of the small group was 64 years. It is tend to occur larger tumor in young age with statistical significance $(p=0.046$ ). The authors, however, believe that this result comes from small number of cases. In other words, the authors cannot conclude that there is definite relationship between tumor size and age group.

No severe or permanent neurological deficits, such as paralysis or hypesthesia, occurred in this study. In a single case (case number 16), a patient with a very large tumor $(11.6 \times 3.0 \times$ $3.1 \mathrm{~cm}$ ) noted mild motor weakness on the ipsilateral side postoperatively. However, this symptom disappeared gradually over a week, and one month after surgery the patient reported no difficulties with activities of daily living.

If a large tumor or tumor with heavy adhesions is shown on preoperative MRI, IONM is useful to prevent iatrogenic nerve injury during surgery. In this study, the authors utilized IONM in 17 of 18 cases. The authors suppose that this high rate of utilization of IONM contributed to the low rate of 
complications. EMG is the recoding of electrical activity of muscle. Changes in EMG recordings during surgery imply the possibility of nerve injury. So IONM using can assure integrity or injury of nerves.

There are a few limitations in this study. First, this study is limited by the relatively small number of patients. However, if the number of cases was increased, statistically significant results could be expected. Second, brachial plexus tumors have three traditional surgical approaches : anterior supraclavicular approach, anterior infraclavicular approach, and posterior subscapular approach. In this study, however, only the supraclavicular and infraclavicular approaches were reported, and the subscapular approach was not used.

\section{CONCLUSION}

Surgical resection of the tumor is the treatment of choice in the majority of benign and malignant BPTs. For a successful surgical outcome, an appropriate surgical approach is essential, and the appropriate surgical approach is determined by the tumor location and size. Intraoperative nerve injury may occur during surgery. Applying IONM may prevent postoperative complications and it is favorable option for BPTs surgery. Furthermore, abundant direct and indirect surgical experience is important factor that can produce favorable surgical outcome.

\section{CONFLICTS OF INTEREST}

No potential conflict of interest relevant to this article was reported.

\section{INFORMED CONSENT}

Informed consent was obtained from all individual participants included in this study.

\section{References}

1. Binder DK, Smith JS, Barbaro NM : Primary brachial plexus tumors: imaging, surgical, and pathological findings in 25 patients. Neurosurg Focus 16 : E11, 2004

2. Burger $P C$, Scheithauer BW, Vogel FS : Surgical pathology of the nervous system and its coverings, ed 4 . New York: Churchill Livingstone, 2002

3. Crutcher CL 2nd, Kline DG, Tender GC : A modified, less invasive posterior subscapular approach to the brachial plexus: case report and technical note. Neurosurg Focus 42 : E7, 2017

4. Das S, Ganju A, Tiel RL, Kline DG : Tumors of the brachial plexus. Neurosurg Focus 22 : E26, 2007

5. Dubuisson AS, Kline DG, Weinshel SS : Posterior subscapular approach to the brachial plexus. Report of 102 patients. J Neurosurg 79 : 319330, 1993

6. Ellison D, Love S, Chimelli LMC, Harding B, Lowe JS, Vinters HV, et al. : Neuropathology E-Book: A Reference Text of CNS Pathology, ed 3. Edinburgh : Mosby Elsevier, 2013

7. Ganju A, Roosen N, Kline DG, Tiel RL : Outcomes in a consecutive series of 111 surgically treated plexal tumors: a review of the experience at the Louisiana State University Health Sciences Center. J Neurosurg 95 : 51-60, 2001

8. Kim DH, Murovic JA, Tiel RL, Moes G, Kline DG : A series of 146 peripheral non-neural sheath nerve tumors: 30-year experience at Louisiana State University Health Sciences Center. J Neurosurg 102 : 256-266, 2005

9. Lwu S, Midha R : Clinical examination of brachial and pelvic plexus tumors. Neurosurg Focus 22 : E5, 2007

10. Tschoe C, Holsapple JW, Binello E : Resection of primary brachial plexus tumor via a modified supraclavicular approach. J Neurol Surg Rep 75 : e133-e135, 2014 